\title{
VALORES ANUAIS ABSOLUTOS DE SÉRIES DIÁRIAS DE TEMPERATURA MÁXIMA E MÍNIMA DO AR NO ESTADO DE SÃO PAULO: TENDÊNCIAS, PERIODICIDADES E PROBABILIDADES ASSOCIADAS
}

\author{
GABRIEL CONSTANTINO BLAIN ${ }^{1}$, JORGE LULU² \\ ${ }^{1}$ Instituto Agronômico (IAC), Campinas, SP, Brasil \\ ${ }^{2}$ Companhia Nacional de Abastecimento (CONAB), Brasília, DF, Brasil \\ gabriel@iac.sp.gov.br, j_lulu@yahoo.com \\ Recebido Junho 2009 - Aceito Abril 2010
}

\begin{abstract}
RESUMO
Devido a importância do conhecimento da probabilidade de ocorrência de valores anuais absolutos de temperatura máxima (Tmaxabs) e mínima (Tminabs) do ar, o objetivo do trabalho foi caracterizar séries temporais de Tmaxabs e Tminabs, investigando possíveis tendências climáticas, periodicidades e probabilidades de ocorrência associadas aos dados no Estado de São Paulo. Por meio de métodos não paramétricos e espectrais, verificou-se a inexistência de tendências e periodicidades presentes nas séries, excluindo-se a da localidade de Ubatuba relativa à Tminabs. A função associada à distribuição Geral dos Valores Extremos e à distribuição normal podem ser utilizadas, respectivamente, para a estimação das probabilidades de ocorrência dos valores de Tmaxabs e Tminabs. A análise de ondaleta indicou concomitância entre os picos de potência mais elevados dos sinais de Tmaxabs e os casos mais severos de seca meteorológica registrados na literatura científica do Estado de São Paulo. A mesma concomitância não foi observada nas séries de Tminabs.
\end{abstract}

Palavras-Chave: tendências climáticas, probabilidade de ocorrência

\begin{abstract}
ABSOLUT DAILY VALUES OF ANNUAL MAXIMUM AND MINIMUM AIR TEMPERATURE SERIES OF THE STATE OF SÃO PAULO, BRAZIL: TRENDS, PERIODICITIES AND ASSOCIATED PROBABILITIES

The aim of this work was to characterize annual series of absolute daily value of maximum (Tmaxabs) and minimum (Tminabs) air temperature in the State of São Paulo, Brazil, detecting possible trends, periodicities and, associated probabilities. Using non parametric methods and spectral analysis, no trends and periodicities were detected in all analyzed series, excluding the Tminabs series of Ubatuba$\mathrm{SP}$, where increasing trends was detected. The probability density function associated to the General Value Distribution and to the normal distribution can be used, respectively, to estimate the probability associated to the Tmaxabs and Tminabs values in all series. The wavelet power spectrum shows appreciable power concentrated within the most severe event of meteorological drought observed in the scientific literature of the State of São Paulo. The same feature was not observed in the Tminabs series. Keywords: Climate trends, probability of occurrence
\end{abstract}




\section{INTRODUÇÃO}

Entre as principais preocupações relativas ao aquecimento global, a intensificação ou a alteração da freqüência de ocorrência de dias com temperaturas atmosféricas máximas e mínimas extremas, ocupam posição de destaque devido aos severos impactos que esses eventos apresentam em todos os ecossistemas terrestres. Segundo Tebaldi et al. (2006), uma das mais importantes implicações das mudanças climáticas são seus efeitos potenciais na variabilidade do clima e, consequentemente, nos eventos extremos.

Trabalhos como os de Karl et al. (1999) e Manton et al. (2001), são exemplos de esforços da comunidade internacional voltados a investigação desse tema. Karl et al. (1999), utilizando dados de diversas partes do globo, indicam tendência global de elevação no número de dias "excessivamente quentes" (extremely hot days) observados em cada ano. Resultados semelhantes a esses foram apontados por Mantan et al. (2001), com base em dados de 15 países do sudeste asiático e do sudeste do Oceano Pacífico. Mais recentemente, Alexander et al. (2006) analisando dados globais extremos de temperatura do ar e precipitação pluvial, na escala diária, identificaram significativa elevação nas temperaturas noturnas em $70 \%$ das regiões analisadas. Em estudos regionais da temperatura do ar do Continente Sul Americano, Vincent et al. (2005) observaram tendências de elevação principalmente ligadas aos dados noturnos. Segundo esses autores, as alterações nas séries temporais das temperaturas diurnas tiveram significância e coerência espacial (spatial coerency) consideravelmente inferior às verificadas na temperatura noturna.

Sob o ponto de vista de intensificação dos valores extremos absolutos de temperatura do ar, precipitação pluvial e velocidade do vento na localidade de Piracicaba, Estado de São Paulo, Sansigolo (2008) afirma que as séries temporais dessas variáveis, entre 1917 a 2007, não apresentaram tendências significativas de elevação ou queda. Ainda segundo esse autor, a teoria dos valores extremos (TVE), desenvolvida por Fisher e Tippett (1928), é fundamental para a modelagem do risco de ocorrência de eventos raros ou extremos. Fisher e Tippett (1928) definiram três casos especiais de distribuição paramétrica, conhecidos como Gumbel (tipo I), Fréchet (tipo II) e Weibull (tipo III) (Gumbel, 1958), formas particulares da Distribuição Generalizada de Valores Extremos (GEV) desenvolvida por Jenkinson (1955). Para Raynal (1997), um problema que surge na prática, é o da escolha de qual tipo é o mais adequado à amostra sob investigação, sugerindo como alternativa a utilização da GEV. Segundo Nadarajah e Choi, (2007), a GEV possui toda a flexibilidade contida em seus casos particulares.

Considerando a importância do conhecimento do risco de ocorrência de valores anuais absolutos de temperatura máxima (Tmaxabs) e mínima (Tminabs) do ar, bem como da investigação de possíveis alterações temporais nos valores dessas variáveis, o objetivo do trabalho foi caracterizar séries temporais de Tmaxabs e Tminabs do Estado de São Paulo, investigando possíveis tendências, periodicidades e probabilidades associadas aos dados.

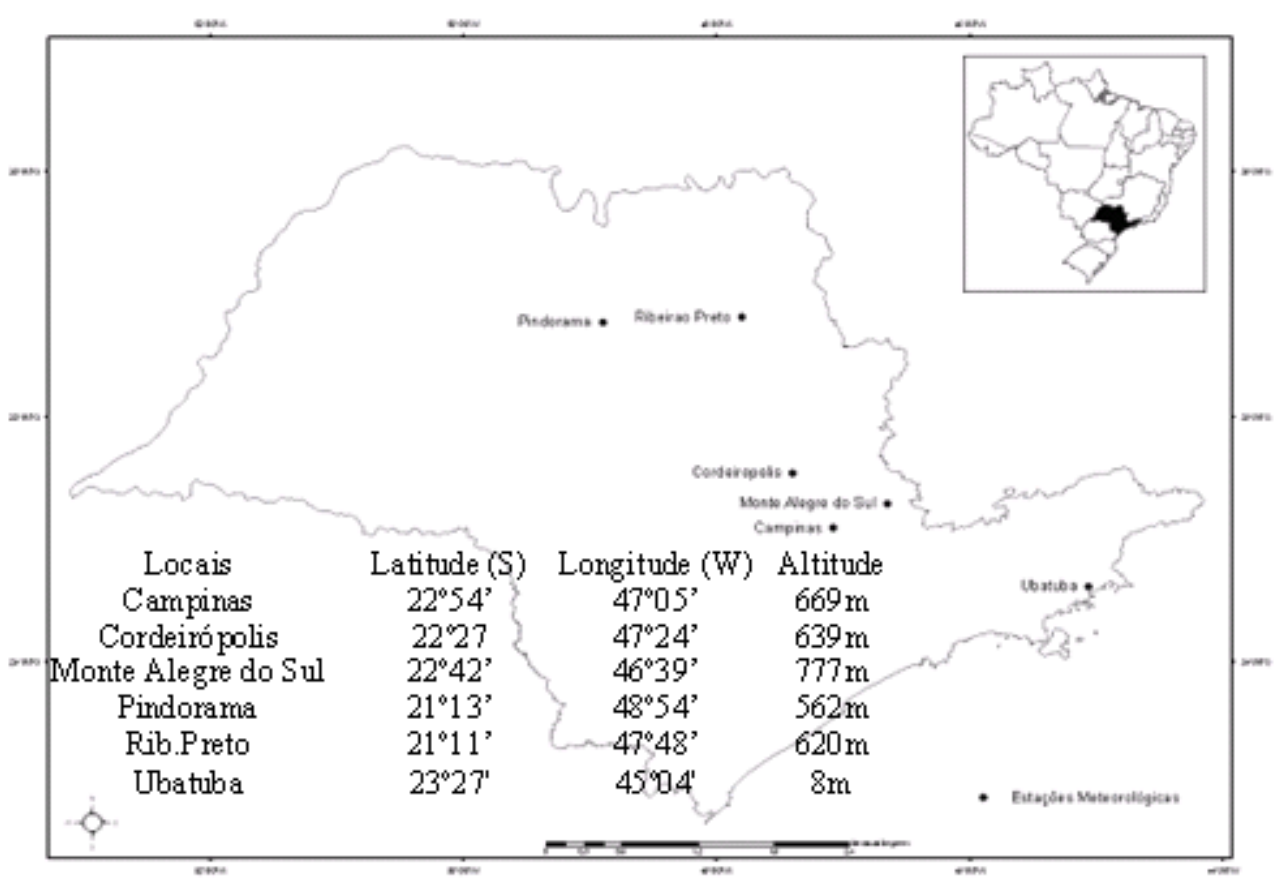

Figura 1 - Postos meteorológicos pertencentes à Secretaria e Agricultura e Abastecimento do Estado de São Paulo utilizados no estudo. 


\section{MATERIAL E MÉTODOS}

Foram utilizados dados anuais absolutos de temperatura máxima e mínima pertencentes ao Instituto Agronômico da Secretaria de Agricultura e Abastecimento do Estado de São Paulo (IAC/SAA-SP). Na Figura 1 são ilustradas as coordenadas geográficas dos postos utilizados. Com exceção das localidades de Pindorama (1951 a 2007) e Ubatuba (1955 a 2007), todas as demais séries estão contidas no período comum de 1948 a 2007.

A função densidade de probabilidade da GEV é descrita por:

$f(x)=\frac{1}{\beta}\left[1+\frac{k(x-\zeta)^{-1-\frac{1}{k}}}{\beta}\right] \exp \left\{-\left[1+\frac{k(x-\zeta)}{\beta}\right]^{-\frac{1}{k}}\right\}$

para $1+\frac{k(x-\zeta)}{\beta}>0$

Em que: $\zeta, \beta e$ k são, respectivamente, os parâmetros de locação, escala e forma

Conforme descrito, a GEV pode assumir três casos particulares, de acordo com o valor de $\mathrm{k}$. Para $\mathrm{k}=0$, a Equação 1 é denominada distribuição Gumbel ou "Fisher-Tippett Tipo I". Contudo, é provável que devido ao elevado número de trabalhos científicos que a empregam, esse caso particular da GEV é usualmente denominado de a distribuição de valores extremos (Wilks 2006), justificando, com isso, a avaliação de seu ajuste aos dados utilizados. O valor máximo dessa última forma da Equação 1, ocorre quando $x=\zeta$. É importante ressaltar que, segundo Sansigolo (2008) a distribuição Gumbel pode ser ajustada aos dados extremos de temperatura máxima do ar da localidade de Piracicaba.

É importante observar que a TVE exige que os dados sejam: a) independentes, b) oriundos de uma mesma distribuição e c) o número $\mathrm{N}$ de valores que compõem essa série é suficientemente grande para convergir à distribuição empírica à GEV. Contudo, de acordo com Wilks (2006), empiricamente, a GEV é freqüentemente adequada à esse tipo de estudo, mesmo quando nem todas as exigências da TVE são respeitadas. Autores como Madsen et al. (1997) e Wilks (1993) indicam que, nesses casos, a adequação da GEV aos valores observados deve ser realizada em conjunto com outras distribuições. Nesse sentido, ressalta-se que Sansigolo (2008), afirmando que distribuições teóricas de probabilidade são simplesmente funções analíticas, usadas para descrever o comportamento de determinadas variáveis, comprova o ajuste da série de Tminabs da localidade de Piracicaba à distribuição normal. No presente trabalho, além da Equação 1, verificou-se o ajuste das séries de dados absolutas à distribuição normal, conforme descrita em diversos trabalhos, tais como o de Wilks (2006), e ao caso particular da GEV, Gumbel.
Os parâmetros das funções utilizadas foram estimados pelo método da máxima verossimilhança (MV). Segundo Sansigolo (2008), o MV é reconhecido como o mais apropriado para estimação dos parâmetros de uma função densidade de probabilidade. Os testes de aderência qui-quadrado $\left(\chi^{2}\right)$ e Kolmogorov-Smirnov (KS), os gráficos percentil-percentil (PP) e quantil-quantil (QQ), descrito em Wilks (2006), foram utilizados para verificar os ajustes das séries empíricas às funções densidade de probabilidade.

Segundo a nota técnica ${ }^{\circ} 79$ da OMM (1966), o teste de Wald-Wolfowitz (teste Run) consiste em realizar a contagem do número de oscilações dos valores acima e abaixo da mediana de uma série de dados naturalmente ordenada. Esse número de oscilações é chamado de Run. Na condução desse método, deve-se avaliar se o valor observado está dentro da faixa de distribuição considerada normal. Um valor elevado de Run indica muitas oscilações, ao passo que baixos valores indicam um desvio em relação à mediana durante o período de registros. Se a série sob investigação contém N1 e N2 valores inferiores e superiores, respectivamente, a distribuição amostral do número de Runs (NR) total pode, de acordo com o teorema do limite central, ser aproximada pela distribuição normal. Com isso, adotando-se o nível de significância 5\%, esse método, conforme ressaltado por nota técnica $\mathrm{n}^{\circ} 79$ da OMM (1966), indica aleatoriedade ${ }^{1}$ da distribuição temporal dos dados sempre que seu valor final $Z$ estiver contido no intervalo [-1,96:+1,96].

Proposto por Mann (1945) e Kendall e Stuart (1967), o teste não paramétrico de Mann-Kendall (MK) considera como hipótese de nulidade (Ho) a inexistência de tendências monótonas na amostra sob investigação. Valores positivos ou negativos indicam elevação ou queda nos dados da série temporal. Autores como Marengo et al. (2007) utilizam o nível de significância $\alpha=0,05$ para a condução desse método. Adotando esse mesmo nível, Ho não será rejeitada sempre que $-1,96<\mathrm{MK}<1,96$. O teste não paramétrico de Pettitt (Pettitt, 1979) indica se duas amostras, oriundas da mesma série, Y1,...,Yt e Yt+1,...,YT são da mesma população, ilustrando a data aproximada da alteração dos valores da série (changing point). Esse método é uma versão do teste de homogeneidade de populações de Mann-Whitney (teste U). Trabalhos como os de Mortatti et al. (2004) adotam o nível de significância de p= $5 \%$ para sua condução.

Nakken (1998) afirma que a análise de ondaletas é bastante adequada para analisar tendências em séries temporais, revelando mudanças abruptas, pontos de quebra e descontinuidades nos sinais originais, que a análise de Fourier

\footnotetext{
${ }^{1}$ O termo aleatoriedade foi adotado como tradução da palavra inglesas rondomness, utilizada por Thom (1966) na descrição do referido teste. Nesse sentido, o termo não aleatório (non rondomness) pode ser referente à presença de tendências (trends), periodicidades, persistência temporal, entre outras, em uma série temporal.
} 
não é capaz de detectar. O autor afirma também que variáveis meteorológicas requerem, devido à natureza temporal de seus sinais, investigações baseadas no domínio tempo-freqüência, a fim de revelar características espectrais em função do tempo. A ondeleta-mãe apropriada deve ter um padrão semelhante ao sinal em estudo (Nakken, 1998). Nesse sentido, a ondeletamãe de Morlet, assim como os dados de temperatura absoluta, apresenta pontos de máximos, picos superiores, e de mínimos, picos inferiores. Dessa forma, a função ondaleta-mãe utilizada foi a de Morlet. As análises de ondaleta foram aplicadas para todas as séries.

\section{RESULTADOS E DISCUSSÃO}

\subsection{Temperatura máxima absoluta}

Na Tabela 1, são apresentados os resultados da aplicação dos testes MK, Run e de Pettitt nas séries de Tmaxabs, bem como suas médias e desvio padrão.

Por meio dos testes MK e Pettitt, verifica-se a inexistência de tendências monótonas, de elevação ou queda, nos valores de Tmaxabs presentes em todas as séries. O teste Run permaneceu, em todas as localidades, dentro de limite crítico adotado. A análise de ondaleta, para as seis localidades, é ilustrada na Figuras 2.

À exceção da localidade de Ubatuba, a inexistência de periodicidades significativas nas séries de Tmaxabs é indicada pela potência global de ondaleta (GWP, Figura $2 b$ ), que exprime a média temporal para diferentes freqüências da magnitude dos picos de variância do sinal original. Contudo, a potência espectral da ondaleta (WPS, Figura 2a) apresenta potências significativas de Tmaxabs, ocorridas no início da década de 1960 com escalas temporais de 2 a 4 anos em todas as séries. Conforme descrito em diversos trabalhos, tais como o de Blain (2005), nos anos de 1961, 1963 e 1964 foram registrados, no

Tabela 1 - Testes de Pettitt, Mann-Kendall (MK) e Run (Z) aplicados nas séries de temperatura máxima absoluta nas localidades de Campinas, Cordeirópolis, Monte Alegre do Sul, Pindorama, Ribeirão Preto e Ubatuba. A média aritmética, mediana e desvio padrão também são ilustrados.

\begin{tabular}{cccccccc}
\hline & \multicolumn{3}{c}{ Descrição $\left({ }^{\circ} \mathrm{C}\right)$} & \multicolumn{3}{c}{ Testes Estatísticos } \\
\cline { 5 - 7 } Local & Média & Mediana & Desvio Padrão & $Z$ & MK & Pettitt \\
\hline Campinas & 35,1 & 35 & 1,13 & 1,27 & 0,98 & NS \\
& & & & & & & \\
Cordeirópolis & 35,2 & 35,2 & 1,37 & 1,94 & 0,13 & NS \\
& & & & & & & \\
MonteAlegre & 34,6 & 34,4 & 1,26 & $-1,37$ & 0,41 & NS \\
& & & & & & \\
Pindorama & 36,5 & 36,4 & 1,4 & 1,55 & 0,01 & NS \\
RibPreto & 36,6 & 36,6 & 1,28 & 0,58 & 0,72 & NS \\
Ubatuba & 37 & 37,1 & 1,46 & 1,93 & 0,78 & NS \\
\hline
\end{tabular}

Estado de São Paulo, os casos mais severos de seca relatados na literatura científica. Infere-se, portanto, que os maiores valores de Tmaxabs das séries analisadas foram condicionados pelos casos mais intensos de déficit no regime de precipitação pluvial observados no Estado de São Paulo. A justificativa física dessa hipótese repousa no fato de que o baixo teor de umidade no solo desloca o balanço de energia em superfície no sentido do calor sensível, uma vez que o gasto com calor latente é limitado. Dessa forma, há disponibilidade energética para condicionar os maiores valores de temperatura do ar relativos a cada série. Especificamente para a série de Ubatuba, verifica-se que a periodicidade apontada pela GWP é temporalmente localizada apenas nas décadas de 1960 e 1990.

Considerando as análises ilustradas na Tabela $1 \mathrm{e}$ na Figuras 2, observa-se que as exigências da TVE são satisfatoriamente observadas em todas as localidades. Nas Figuras 3 e 4, são ilustrados os gráficos PP e QQ, bem como os valores do teste KS para as séries de Tmaxabs utilizadas.

Os resultados dos testes $\mathrm{KS}$ e $\chi^{2}$ foram concordantes em todos os casos. Ao contrário do $\chi^{2}$, a interpretação do valor final do KS depende apenas do número de dados constituintes de cada série e, evidentemente, do nível de significância adotado. Dessa forma, optou-se apenas pela ilustração dos resultados desse último método. Por meio das análises ilustradas nas Figuras 3 e 4, verificou-se que a função densidade de probabilidade associada à GEV foi a que melhor descreveu as freqüências de ocorrência dos valores de Tmaxabs nas localidades sob investigação no período considerado.

\subsection{Temperatura mínima absoluta}

Na Tabela 2 são ilustradas as médias, medianas e os desvios padrão das seis séries de Tminabs.

Os resultados dos testes MK e de Pettitt, também apresentados, indicam que à exceção da localidade de Ubatuba, as demais séries podem ser consideradas livres de tendências significativas. Para a referida série litorânea, o MK indica tendência significativa de elevação que, de acordo com o teste de Pettitt, tem sua data de inicio aproximado em 1968. Esses últimos resultados são corroborados pelo teste $\mathrm{Z}$, que indica presença de componentes não aleatórias nos dados da localidade de Ubatuba. Na Figuras 5 são ilustradas as análises de ondaleta empregadas no estudo.

Por meio da WPS da Figura 5, nota-se que os picos de variância, ainda que apontados como significativos pela GWP, ocorrem de forma temporalmente esparsa no sinal temporal da Tminabs; sendo, portanto, restritos a determinados segmentos do período analisado. Na localidade de Monte Alegre do Sul, por exemplo, a WPS indica que essas potências estão fundamentalmente contidas nos anos de 1968 a 1982 e 1988 a 
a) U⿺PS - Campinas

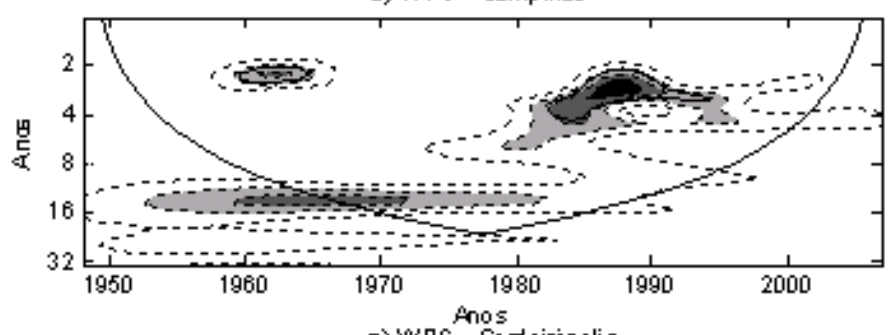

a) UPs - Anos

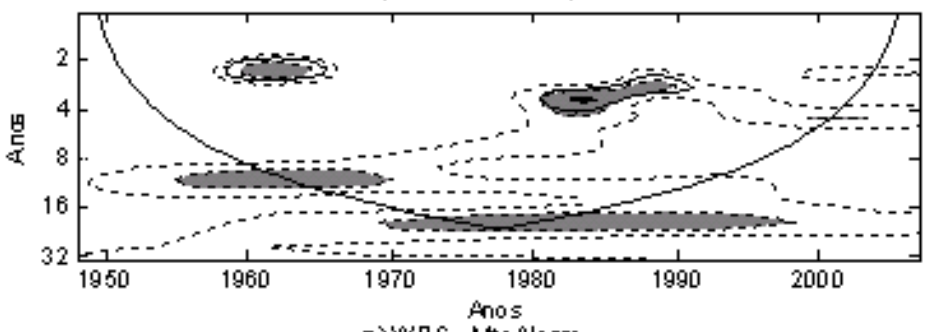

a) W'S - MiteAlegre

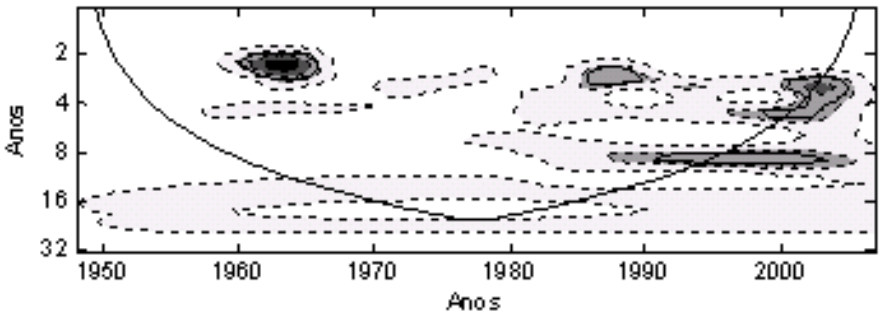

a) U'PS - Pindorama

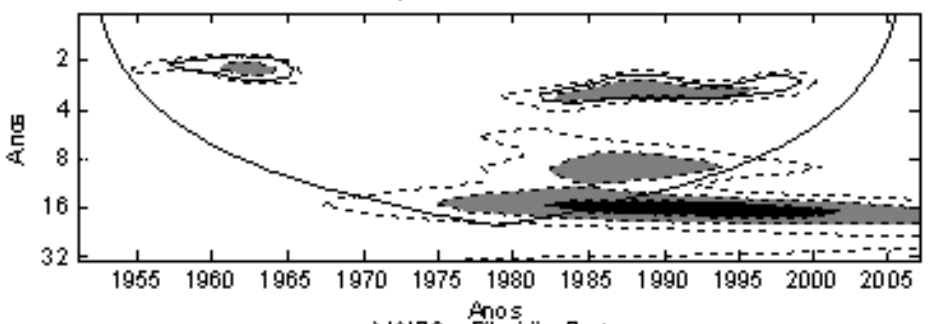

a) UPS - Fibeiräo Preto

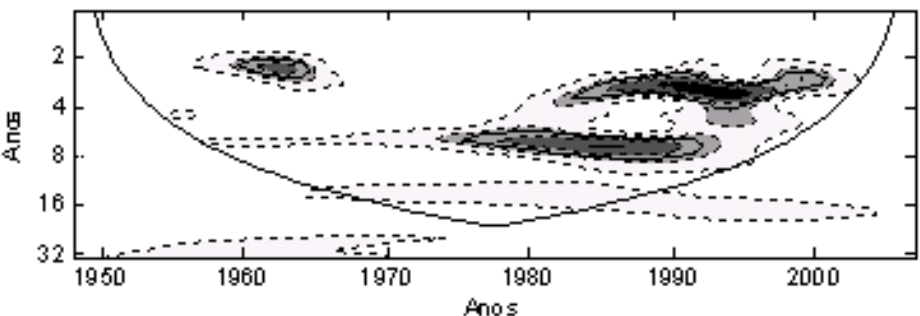

a) Uip $S$ - Ubatuba

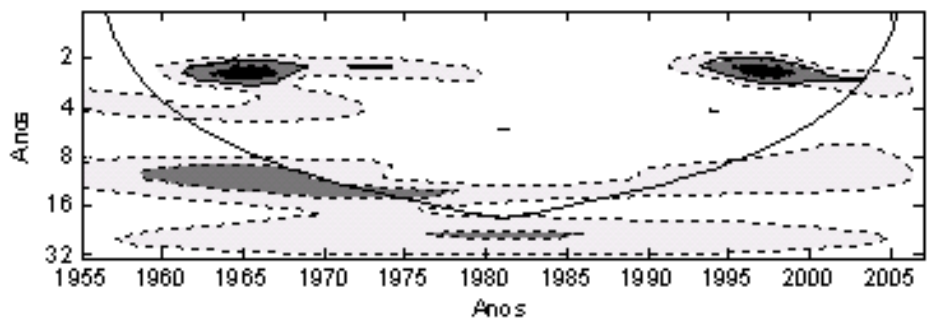

b) GUiP
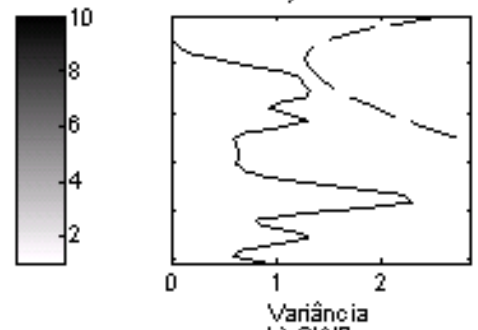

b) Gưp
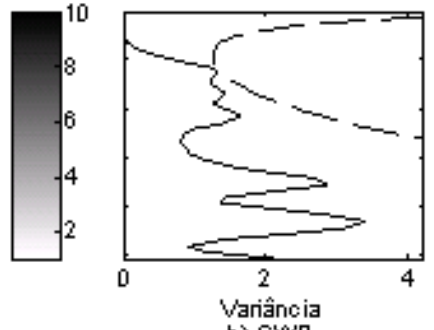

b) GUu'P
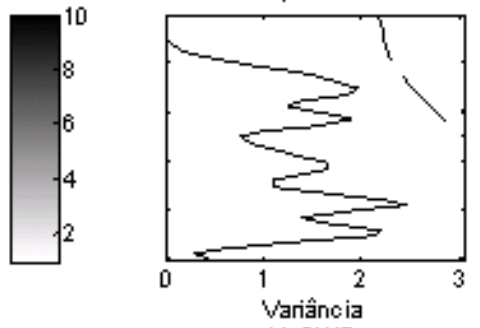

b) Gưp
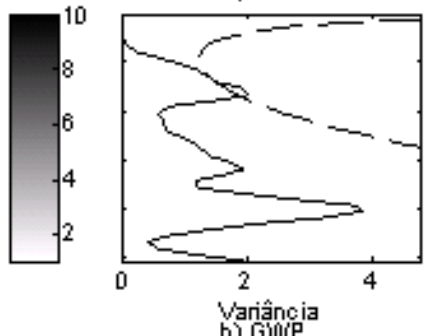

b) Givip
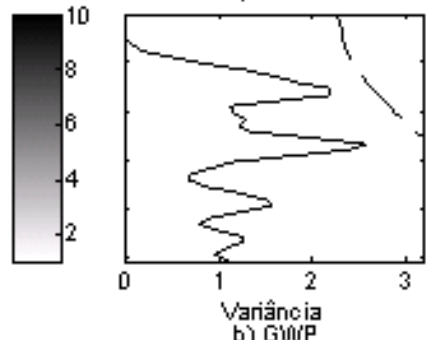

b) Givi?
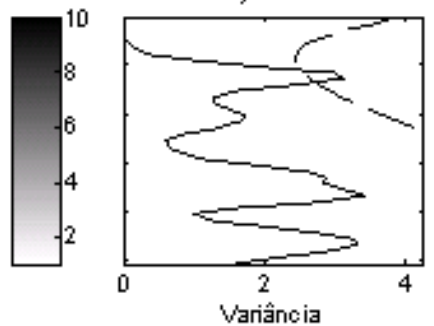

Figura 2 - a) Espectro de energia local da ondaleta da temperatura máxima absoluta. Contornos tracejados correspondem a valores de variância normalizados variando de 1 a 10, (barra horizontal). Contornos sombreados englobam áreas com variâncias significativas ao nível de 95\%. A curva em forma de U representa o cone de influência. b) Espectro global de ondaleta; c) média por escalas das potências observadas. 

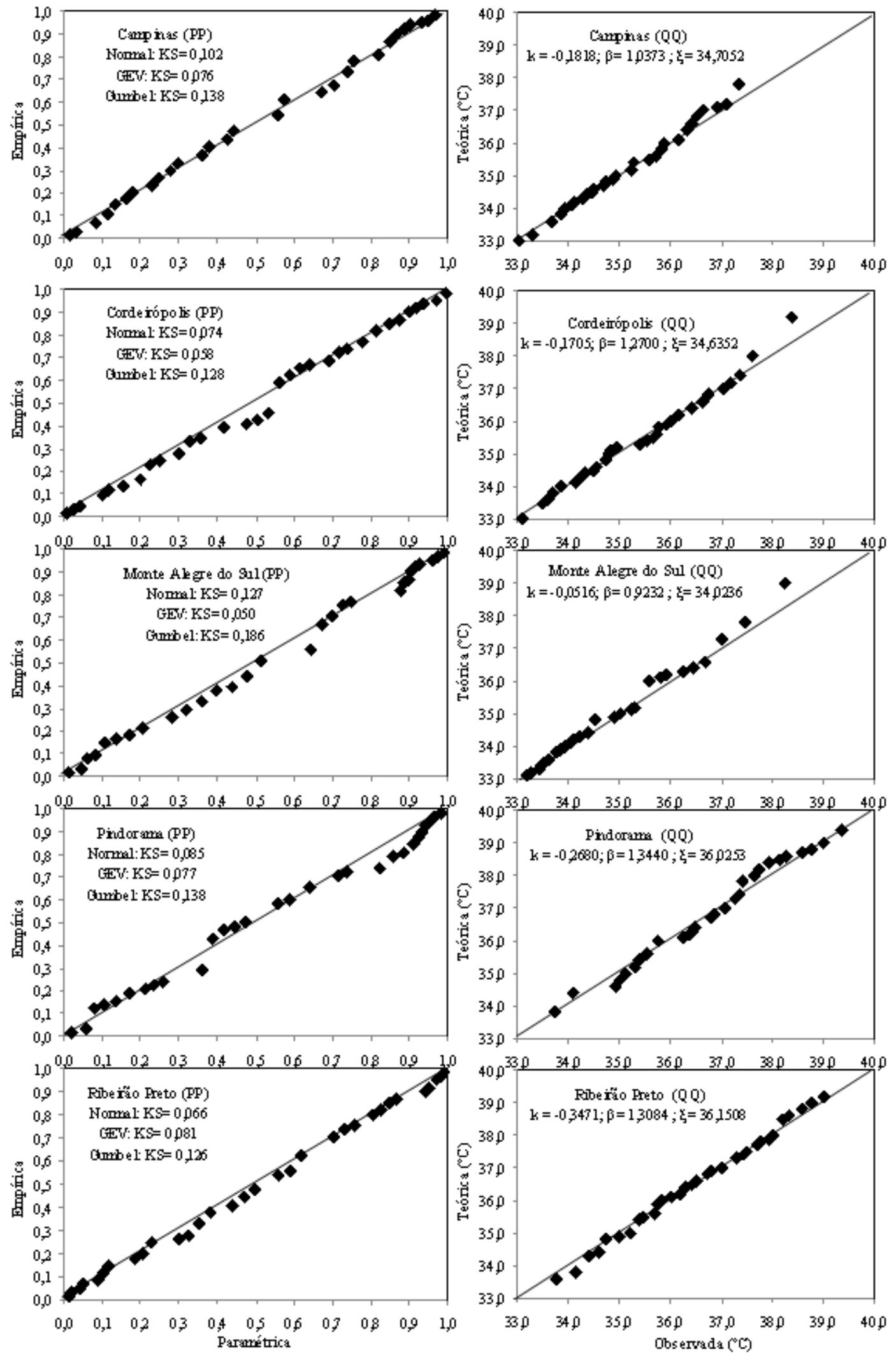

Figura 3 - Gráficos percentil-percentil (PP) e quantil-quantil (QQ) relativos ao ajuste de séries de temperatura máxima absoluta do ar no Estado de São Paulo à distribuição geral de valores extremos (GEV). Os resultados dos testes kolmogorov-Smirnov (KS) para as distribuições normal, gumbel e GEV (com os parâmetros) também são ilustrados. 
Tabela 2 - Testes de Pettitt, Mann-Kendall (MK) e Run (Z) aplicados nas séries de temperatura mímima absoluta nas localidades de Campinas, Cordeirópolis, Monte Alegre do Sul, Pindorama, Ribeirão Preto e Ubatuba. A média aritmética, mediana e desvio padrão também são ilustrados.

\begin{tabular}{ccccccc}
\hline Local & Média & Mediana & Desvio Padrão & Z & MK & Pettitt \\
\hline Campinas & 4,6 & 4,6 & 2,52 & $-0,81$ & 1,64 & NS \\
Cordeirópolis & 2,8 & 3 & 1,99 & 1,93 & 1,21 & NS \\
MteAlegre & 2,7 & 3 & 1,94 & $-1,09$ & 0,15 & NS \\
Pindorama & 3,8 & 3,9 & 2,39 & 0,73 & $-0,52$ & NS \\
RibPreto & 4,1 & 4,5 & 2,54 & 0,82 & 1,94 & NS \\
Ubatuba & 7 & 7 & 1,85 & 2,86 & 3,92 & 1968 \\
\hline
\end{tabular}

1995. Na localidade de Pindorama esses picos são verificados nos anos de 1958 a 1962 e 1973 a 1997, ao passo que na série de Ribeirão Preto são observados no período de 1975 a 1986 e na década de 1990. Nas localidades de Campinas, Cordeirópolis e Ubatuba, a análise de ondaleta não indicou picos significativos de variância.

Ao contrário do observado para a Tmaxabs, não houve marcantes concomitâncias entre os picos de potência local da ondaleta, relativa às séries de Tminabs, e os casos mais severos de seca anteriormente citados. Uma hipótese física que justificaria tal característica é de que, apesar de ocorrer, ao longo desses períodos de intensa seca meteorológica, um deslocamento do balanço de energia no sentido do calor sensível (durante o período diurno), uma atmosfera mais seca também favorece a perda de energia por ondas longas pela superfície. Em outras palavras, infere-se que em épocas de seca, haja uma maior taxa de resfriamento noturno, compensando, ao menos em parte, a elevação da temperatura ao longo do período diurno.

Nas Figuras 6 e 7, são ilustrados os ajustes das séries de Tminabs às três funções densidade de probabilidade propostas. A detecção de tendências de elevação na localidade de Ubatuba, anteriormente observada, diminui, sob o rigor científico, a relevância dessa parametrização, uma vez que as descrições analíticas das probabilidades de ocorrência, associadas aos valores dessas amostras ou períodos, apresentarão considerável grau de incerteza ao serem extrapoladas para demais amostras ou anos futuros. Os resultados de testes $\mathrm{KS}$ e $\chi^{2}$ foram concordantes em todos os casos.

De forma geral, nas seis localidades, a GEV e a distribuição normal apresentam desempenho semelhantes ao descreverem as probabilidades associadas aos valores de Tminabs, sendo, especialmente na série de Ubatuba, ligeiramente superiores à Gumbel. Na série de Ribeirão Preto a GEV e a Gumbel mostraram-se, de forma pouco marcante, superiores à normal. Dessa forma, considerando as indicações de Astolpho et al. (2004) para à série de Tminabs de Campinas (1890 a 2000), de Sansigolo (2008) para a localidade de Piracicaba (1917 a 2006), as indicações das Figuras 6 e 7 e a relativa facilidade da estimação da função densidade de probabilidade associada à distribuição normal, em relação à GEV e a Gumbel, recomenda-se o uso da distribuição normal para o ajuste dos valores de Tminabs nas séries do Estado de São Paulo, embora a GEV possa também ser empregada.

\subsection{Análise comparativa}

Comparando-se as Figuras 2a e 5a, verifica-se que os picos de variância dos sinais temporais das duas variáveis
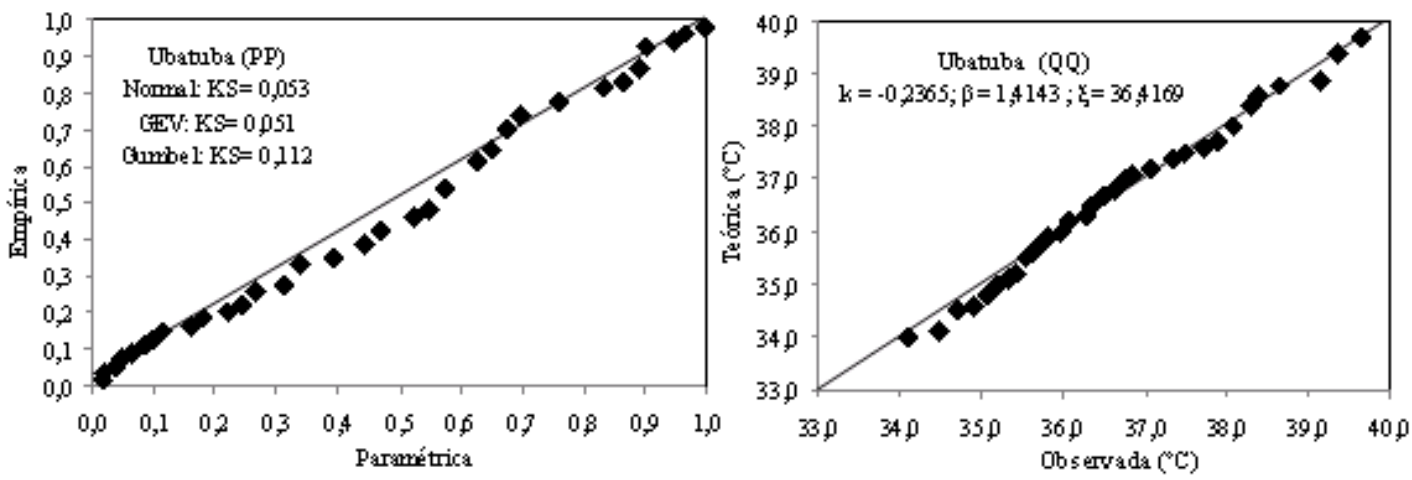

Figura 4 - Gráficos percentil-percentil (PP) e quantil-quantil (QQ) relativos ao ajuste da série de temperatura máxima absoluta do ar na localidade de Ubatuba - Estado de São Paulo - à distribuição geral de valores extremos (GEV). Os resultados dos testes kolmogorov-Smirnov (KS) para as distribuições normal, gumbel e GEV (com os parâmetros) também são ilustrados. 

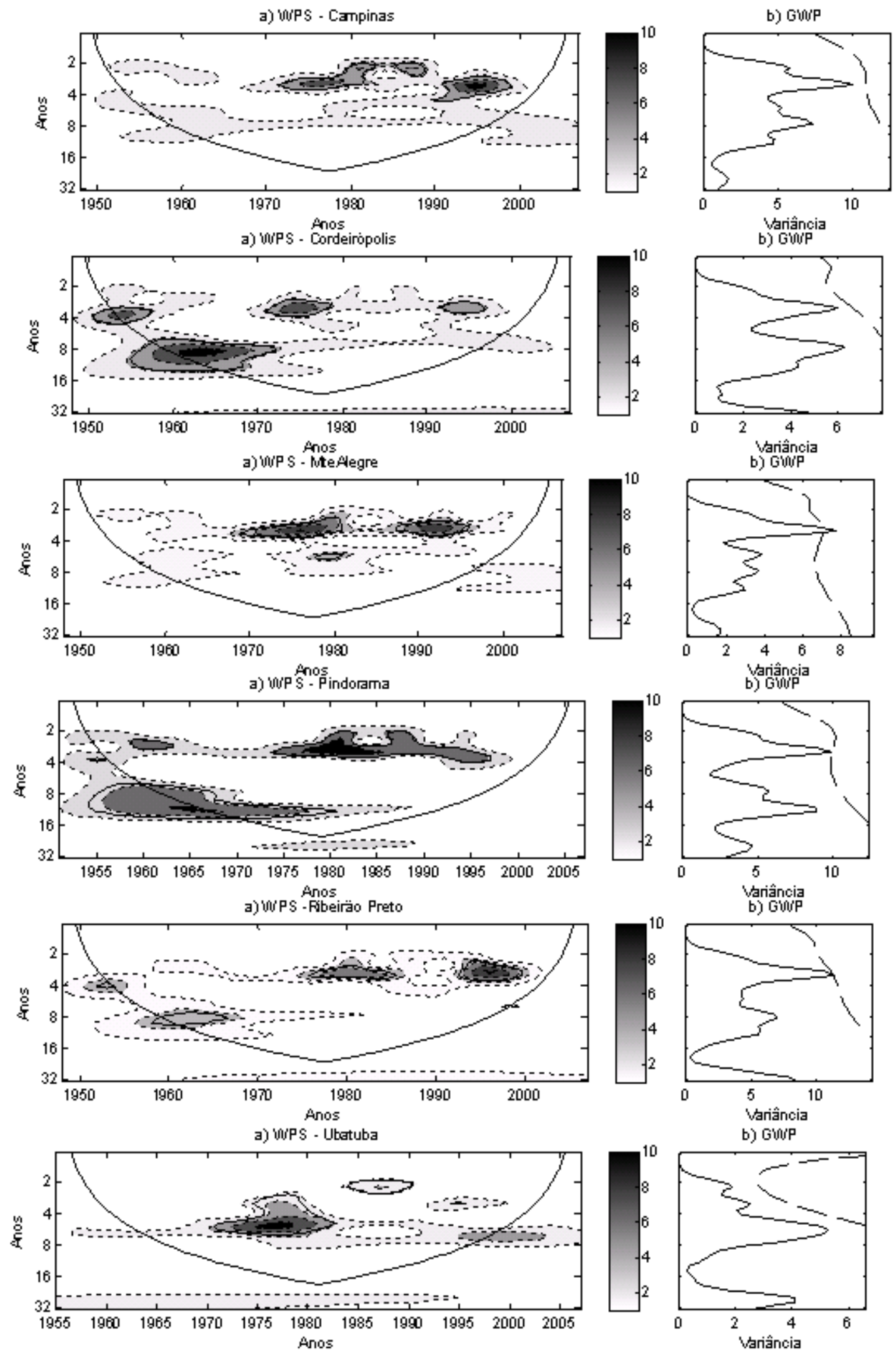

Figura 5 - a) Espectro de energia local da ondaleta da temperatura mínima absoluta. Contornos tracejados correspondem a valores de variância normalizados variando de 1 a 10, (barra horizontal). Contornos sombreados englobam áreas com variâncias significativas ao nível de 95\%. A curva em forma de U representa o cone de influência. b) Espectro global de ondaleta; c) média por escalas das potências observadas. 

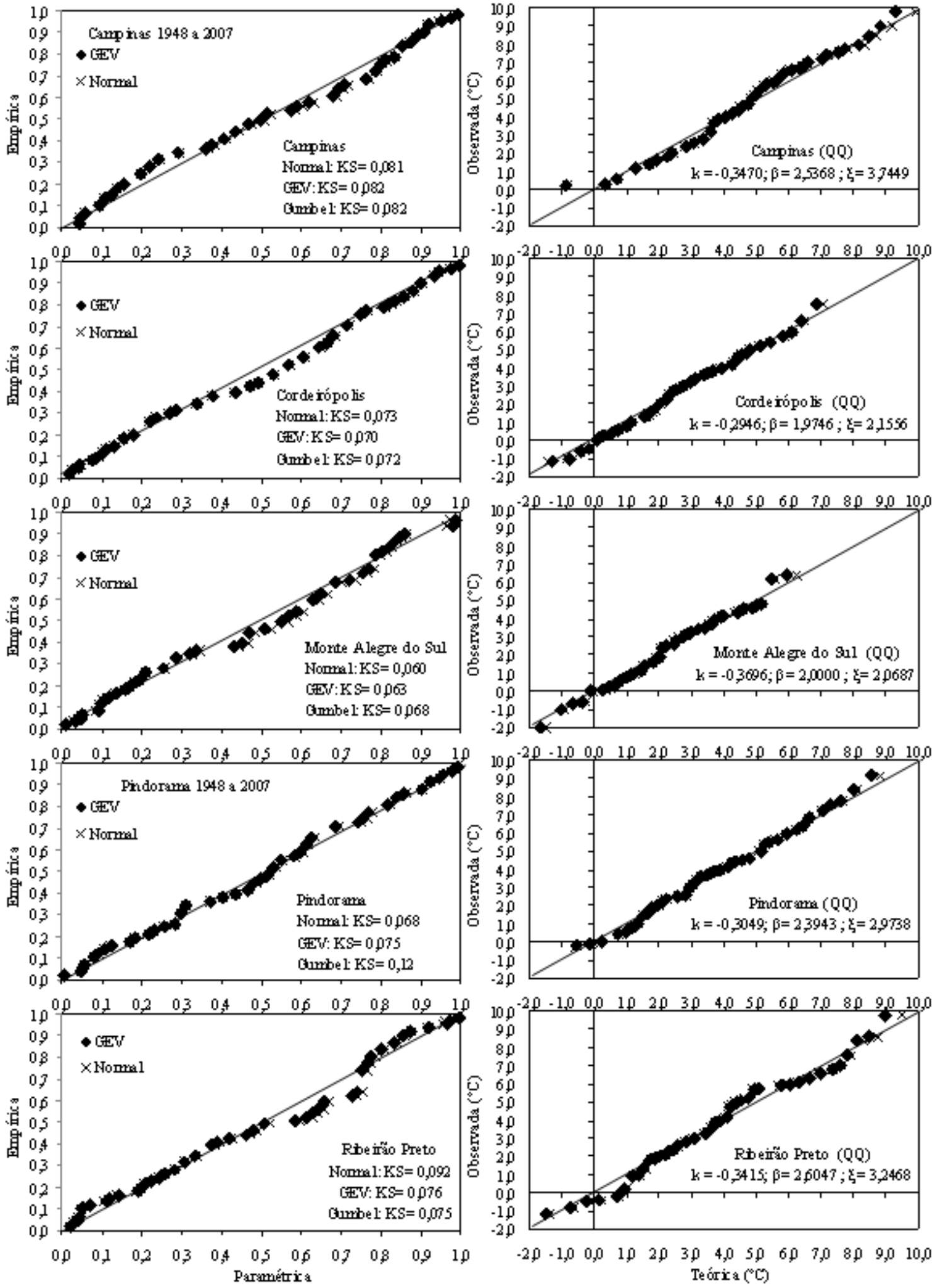

Figura 6 - Gráficos percentil-percentil (PP) e quantil-quantil (QQ) relativos ao ajuste de séries de temperatura mínima absoluta do ar no Estado de São Paulo à distribuição geral de valores extremos (GEV) e a distribuição normal. Os resultados dos testes kolmogorov-Smirnov (KS) para as distribuições normal, gumbel e GEV (com os parâmetros) também são ilustrados. 

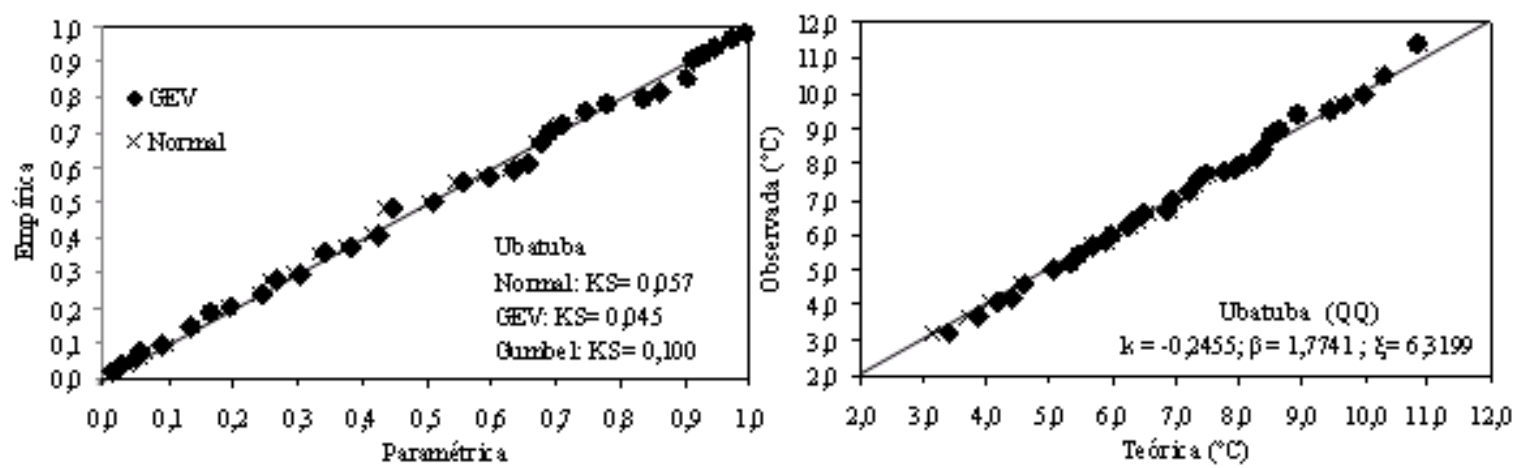

Figura 7 - Gráficos percentil-percentil (PP) e quantil-quantil (QQ) relativos ao ajuste da séries de temperatura mínima absoluta do ar da localidade de Ubatuba no Estado de São Paulo à distribuição geral de valores extremos (GEV) e a distribuição normal. Os resultados dos testes kolmogorovSmirnov (KS) para as distribuições normal, gumbel e GEV (com os parâmetros) também são ilustrados.

analisadas não ocorrem de forma concomitante. Sob o ponto de vista de investigação científica, essa última característica pode ser vista como uma indicação estatística de que as variabilidades das séries temporais de Tminabs e de Tmaxabs são influenciadas de forma diferenciada pelas forçantes moduladoras do clima das regiões analisadas.

Sob o ponto de vista de alterações climáticas, apenas a série de Tminabs da localidade de Ubatuba apresentou tendência de elevação em seus dados. Nesse ponto, é interessante citar as afirmações de Vincent et al. (2005) que indicam que as maiores tendências de elevação, nas séries de temperatura do ar utilizadas por esses autores, foram observadas nas costas leste e oeste da América do Sul. Para as demais localidades, ressalta-se que trabalhos, como os de Blain et al. (2009) e de Blain (2010), indicam significativas tendências de elevação em séries anuais de temperatura média máxima e, especialmente, média mínima do ar no Estado de São Paulo. Contudo, assim como observado por Sansigolo (2008) para a localidade de Piracicaba, essa última característica de elevação não foi significativa na intensidade dos eventos absolutos de temperatura do ar (à exceção da série de Tminabs de Ubatuba). Em outras palavras, infere-se que a influência das forçantes radioativas (escala global ou local) responsável por alterações climáticas como as apontadas por Blain et al. (2009) e Blain (2010), ainda não é significativamente observada nas séries de valores absolutos de temperatura do ar. Ressalte-se que, conforme Sansigolo (2008), essa última afirmação é relativa à intensidade dos eventos extremos e não às suas freqüências de ocorrência que, por sua vez, podem ser afetadas por mudanças de ordem climática.

\section{CONCLUSÃO}

As séries anuais de temperatura máxima diária absoluta das localidades utilizadas não apresentam indícios de tendências climáticas de elevação ou queda em seus valores. Verificou-se o bom ajuste dessas séries à distribuição Geral dos Valores Extremos. Sob o ponto de vista de inferências científicas, a análise espectral da ondaleta indicou concomitância entre os picos de potência dos sinais temporais de Tmaxabs e os casos mais severos de seca observados no Estado de São Paulo, indicando possível modulação dos maiores valores das séries investigadas por intensos déficits no regime de precipitação pluvial.

Para as séries de temperatura mínima absoluta do ar, observou-se na localidade de Ubatuba tendência de elevação com início aproximado no ano de 1968. As demais localidades podem ser consideradas livres de tendência. As funções paramétricas, associadas às distribuições normal e Geral dos Valores Extremos, foram as que proporcionam as melhores descrições analíticas das probabilidades de ocorrência associadas aos valores de Tminabs nas seis localidades analisadas. Ressalta-se, contudo, que a detecção de tendências de elevação nos dados de Ubatuba, diminui a relevância dessa parametrização. Não houve marcantes concomitâncias entre os picos de potência local da ondaleta, relativa às séries de Tminabs, e os casos mais severos de seca registrados no Estado de São Paulo.

O resultado da análise espectral aplicada permitiu também inferir que: i) as variabilidades das séries temporais, de Tminabs e de Tmaxabs, são influenciadas de forma diferenciada pelas forçantes moduladoras do clima das regiões analisadas e, ii) que a influência de fatores, de escala global ou local, responsável por possíveis alterações climáticas ainda não é significativamente observada nas séries de valores absolutos de temperatura do ar das localidades de Campinas, Cordeirópolis, Monte Alegre do Sul, Pindorama e Ribeirão Preto.

\section{REFERÊNCIAS BIBLIOGRÁFICAS}

ALEXANDER, L. V.; ZHANG, X.; PETERSON, T. C.; CAESAR, J.; GLEASON, B.; TANK, A. M. G; HAYLOCK, 
M.; COLLINS, D.; TREVIN, B.; RAHIMZADEH, F.; TAGIPOU, A.; RUPA KUMAR, K.; REVADEKAR, J.; GRIFFITHS, G.; VINCENT, L.; STEPHENSON, D.; BURN, J.; AGUILLAR, E.; TAYLOR, M.; NEW, M.; ZHAI, P.; RUSTICUCCI, M.; VASQUEZ-AGUIRRE, J. L. Global observed changes in daily climate extremes of temperature and precipitation. J. Geophys. Res., v.111, D05109, 2006.

AStOlPhO, F.; CAMARGO, M. B. P.; BARDin, L. Probabilidades mensais e anuais de ocorrência de temperaturas mínimas do ar adversas à agricultura na região de Campinas (SP), de 1891 a 2000. Bragantia, v.63, n.1, p.141-147, 2004

BLAIN, G, C, Avaliação e adaptação do Índice de Severidade de Seca de Palmer (PDSI) e do Índice Padronizado de Precipitação (SPI) às condições climáticas do Estado de São Paulo, 2005, 120p, Dissertação (Mestrado em Agricultura Tropical e Sub-tropical) - Instituto Agronômico, Campinas - SP.

BlAin, G, C, PICOLI, M., C., A., LUlu, J., Análises estatísticas das tendências de elevação nas séries anuais de temperatura mínima do ar no Estado de São Paulo. Bragantia, v.68, n.2, p.423-433, 2009.

BLAIN, G, C, Séries anuais de temperatura máxima média do ar no Estado de São Paulo: variações e tendências climáticas. Revista Brasileira de Meteorologia (prelo)

FISCHER, R.A., TIPPETT, L.H.C., Limiting forms of the frequency distribution of the largest or smallest member of a sample. Proceeding of the Cambridge Philosophical Society, v.14, p. 180-190, 1928

GUMBEL, E. J. Statistics of Extremes. Columbia University Press, New York, 375p. 1958.

JENKINSON, A. F. The frequency distribution of the annual maximum (or minimum) values of meteorological elements. Quarterly Journal of the Royal Meteorological Society, v. 81, p. 159-171, 1955.

KARL, T.; R.; NICHOLLS, N.; GHAZI, A.; Workshop on indices and indicators for climate extremes. Climatic Change. CLIVAR/GCOS/WMO v.42, p. 3-7, 1999

KENDALL, M.A.; STUART,A. The advanced theory of statistics. Londres: Charles Griffin \& Company Limited, 1967. 690p. MADSEN, H.; RASMUSSEN, P. F.; ROSBJERG, D. Comparison of Annual Maximum Series and Partial Duration Series Methods for Modeling Extreme Hydrologic Events 1. At-Site Modeling, Water Resource Research, v.33, n.4, p.747-757, 1997

MANN, H.B., Non-parametric tests against trend, Econometrica 13, MathSciNet, 1945, pp. 245-259.

MANTON, M.;J;; DELLA-MARTA, P.; M.; HAYLOCK, M.;R.; HENNESSY, K.;J.; NICHOLLS, N.; CHAMBERS, L.;E.;
COLLINS, D.;A.; DAW, G.; FINET, A.; GUNAWAN, D.; INAPE, K.; ISOBE, H.; KESTIN, T.;S.; LEFALE, P.; LEYU, C.;H.; LWIN, T.; MAITREPIERRE, L.; OUPRASITWONG, N.; PAGE, C.;M.; PAHALAD, J.; PLUMMER, N.; SALINGER, M.;J.; SUPPIAH, R.; TRAN, V.;L.; TREWIN, B.; TIBIG, I.; YEE, D.; Trends in extreme daily rainfall and temperature in Southeast Asia and the South Pacific: 1961-1998. International Journal of Climatology v. 21, n.3, p. 269-284, 2001

MARENGO, J.; NOBRE, C.; RAIGOZA, D.; VALVERDE, M.; PISNITCHENKO, I.A.; OLIVEIRA, J.C.M. Boletim do Projeto: uso de cenários de mudanças climáticas regionais em estudos de vulnerabilidade e adaptação no Brasil e na América do Sul (GOF-UK-CPTEC), 2007. Disponível em: $<$ www.cptec.inpe.br/mudancas_climaticas $>$. Acesso em: 1 nov. 2008

MORTATTI, J., BORTOLETTO Jr., M., J., MILDE, L.,C.,E., PROSBST, J., Hidrologias dos rios Tietê e Piracicaba: Séries temporais de vazão e hidrogramas de cheia. Revista Ciência e Tecnológica. V.12, n.23, p.55-67. 2004

NADARAJAH, S., CHOI, D., Maximum daily rainnfall in South Korea. Journal of Earth System Science, Índia, v116, n4, p. 311-320, 2007

NAKKEN, M. Wavelet analysis of rainfall-runoff variability isolating climatic from anthropogenic patterns. Environmental Modelling \& Software, v. 14, p. 283-295, 1998. <doi:10.1016/S1364-8152(98)00080-2>

ORGANIZAÇÃO METEOROLÓGICA MUNDIAL: Climatic Change. WMO, Geneva, Switzerland, 80p. 1966.

PETTITT, A.N. A non-parametric approach to the change-point problem. Appllied Statistic, v.28, n. 2, p.126-135, 1979.

RAYNAL, J.A., Sobre el uso del dominio de atracción para la identificación de valores extremos para máximos. Ingenieria Hidrólica en Mexico, v.12, p.57-62, 1997

SANSIGOLO, C., A., Distribuições de extremos de precipitação diária, temperatura máxima e mínima e velocidade do vento em Piracicaba, SP (1917-2006), Revista Brasileira de Meteorologia, v.23, n.3, p. 341-346, 2008, São José dos Campos

TEBALDI, C., HAYHOE, K., ARBLASTER, J., M., MEEHL, G., A., Climate change, v.79, p.185-211, 2006, doi < 10.1007/s10584-006-9051-4>

VINCENT, L.A.; PETERSON, T.C.; BARROS, V.R.; MARINO, M.B.; RUSTICUCCI, M.; CARRASCO, G.; RAMIREZ, E.; ALVES, L.M.; AMBRIZZI, T.; BERLATO, M.A.; GRIMM, A.M.; MARENGO, J.A.; MOLION. L.; MONCUNILL, D.F.; REBELLO, E.; ANUNCIAÇÃO, Y.M.T.; QUINTANA, J.; SANTOS, J.L.; BAEZ, J.; CORONEL, G.; GARCIA, J.; TREBEJO, I.; BIDEGAIN, M.; HAYLOCK, M.R.; KAROLY, D. Observed trends in 
indices of daily temperature extremes in South America 1960-2000. Journal of Climate, Zurich, v.18, p.5011-5023, 2005.

WILKS, D. S. Comparison of Three-Parameter Probability Distributions for Representing Annual Extreme and Partial
Duration Precipitation Series, Water Resource Research, v.29, n.10, p.3543-3549, 1993

WILKS, D.S. Theoretical probability distributions. In: Statistical methods in the atmospheric sciences. San Diego: Academic Press, 2006. p. 102-104. 